\section{Clobazam and its use in epilepsy}

\author{
Marius Pernea, Alastair G. Sutcliffe \\ Department of Pediatrics, Institute of \\ Child Health, University College London, \\ UK
}

\section{Abstract}

Clobazam (CLB) is an older anti-epileptic drug, with a slightly different chemical structure from that of the classic benzodiazepines currently used in the treatment of epilepsy, which confers less sedative properties in terms of negative adverse effects. It is also thought to be better tolerated than other anti-epileptic drugs, whilst maintaining a very similar level of efficacy. It has been tested extensively in over 50 studies on more than 3000 patients with epilepsy and is now approved as an adjunctive treatment of epilepsy in $>100$ countries. The aim of this review is to evaluate several existing studies on the effectiveness of CLB as an adjunctive therapy in the treatment of epilepsy and whether this therapy is more useful in particular types of epilepsy or seizure prevention. This is not a systematic review but a general overview of some of the most recent studies on the effectiveness of CLB as an adjunctive therapy. Additionally, the benefits of having an oral suspension of CLB will be evaluated with regards to patient groups benefiting from this formulation. The last issue addressed is that of the importance of prescribing CLB by brand, along with the benefits and risks of not doing so.

\section{The use of clobazam as an adjunct therapy in the treat- ment of epilepsy}

Clobazam (CLB) is a 1,5-benzodiazepine that has been introduced in 1975 as an anxiolytic drug and shortly after, 1,2 it was discovered that it has strong anti-epileptic properties as well. It is distinguished from other classic 1,4-benzodiazepines in that its nitrogen atoms are placed in $1^{\text {st }}$ and $5^{\text {th }}$ positions in the $B$ ring. Its mechanism of action is similar to other benzodiazepines however CLB is believed to be a partial agonist rather than non-selective full receptor agonists, which is what 1,4-benzodiazepines are. ${ }^{3}$ Moreover, CLB has lower affinity for the $\mathrm{GABA}_{\mathrm{a}}$ subunits and greater selectivity for $a_{2}$ subunits over $a_{1}$ subunits, than the 1,4-benzodiazepines and it is thought that these properties confer to CLB less sedative effects than other benzodiazepines. ${ }^{2,4}$
The standard treatment for epilepsy involves using a single anti-epileptic drug at the minimally effective dose, up to the maximum tolerated dose. ${ }^{5}$ However, the numerous seizure types that a patient may experience render treatments with one agent ineffective so combination therapy is often required. Breakthrough seizures are often experienced by patients; hence continuous adjustments need to be made to their medications regimes over the course of their lifetime, both in terms of dosage and number of agents used. ${ }^{2}$

Due to its less sedative effects and its very similar effectiveness in comparison with other agents, CLB is very frequently selected as an add-on agent when polytherapy is needed, particularly in the case of intractable epilepsy.

Several studies have shown that CLB is an effective adjunctive anti-epileptic drug (AED) for a few specific types of epilepsy, most importantly Lennox-Gastaut syndrome (LGS). These include both retrospective studies and more importantly randomized, double-blind studies.

A randomized, double-blind, dose-ranging study evaluated safety and efficacy of CLB as adjunctive therapy for drop seizures in patients with LGS. $^{6}$ LGS is an epileptic encephalopathy characterized by multiple types of seizures and developmental delay. The presence of a characteristic triad described typical LGS: i) tonic axial, atonic, and/or atypical absence seizures; ii) electroencephalography (EEG) abnormalities with bursts of diffuse slow spike-wave pattern of 1.5-2.5 Hz; and iii) impaired intellectual growth. Atonic or drop seizures are frequent in patients with LGS and are responsible for most injuries associated with falls. Seizures in LGS are refractory to most AEDs hence the need for combinational therapy. 6

The study conducted was a phase II, randomized, double-blind, dose-ranging multicenter study which comprised a 4-week baseline period, a 3-week titration period and a 4-week baseline period. ${ }^{6} \mathrm{~A}$ daily seizure diary record was used to record the number of seizures, specifically any drop seizures. They were recorded as single drop seizures (defined as a drop seizure occurring 15 min or more before and after the next seizure or cluster) or as clusters (defined as two or more drop seizures, with less than 15 min between any two consecutive seizures); non-drop seizures have also been recorded but on a smaller number of patients. Six weight groups have been defined and patients with two or more drop seizures per week during the baseline period were placed in one of six weight groups and randomly assigned to either low-dose CLB (target dose of $0.25 \mathrm{mg} / \mathrm{kg} /$ day; maximum $10 \mathrm{mg} /$ day if weight was lower than $37.6 \mathrm{~kg}$ ) or high-dose CLB (target dose of $1.0 \mathrm{mg} / \mathrm{kg} /$ day; maximum $40 \mathrm{mg} /$ day if weight was higher than $37.6 \mathrm{~kg}$ ). A significant reduction in drop seizure rates
Correspondence: Correspondence: Alastair G. Sutcliffe, GAP Unit, Institute of Child Health, University College London, 30 Guilford Street, London, WC1N 1EH, UK.

Tel.: +44.020.790.52190 - Fax +44.020.790.52834. E-mail: a.sutcliffe@ucl.ac.uk

Key words: Clobazam; adjunctive therapy; generic prescribing; oral suspension.

Contributions: the authors contributed equally.

Funding: Martindale Pharma provided financial support and reviewed the manuscript for accuracy.

Conflict of interest: the authors declare no potential conflict of interest.

Received for publication: 21 March 2016.

Revision received: 18 April 2016.

Accepted for publication: 22 April 2016.

This work is licensed under a Creative Commons Attribution NonCommercial 4.0 License (CC BYNC 4.0).

(C) Copyright M. Pernea and A. G. Sutcliffe, 2016 Licensee PAGEPress, Italy

Pediatric Reports 2016; 8:6516

doi:10.4081/pr.2016.6516

was observed both in the low-dose group (mean reduction $=12 \% ; \mathrm{P}=0.0162$ ) and in the high-dose group (mean reduction $=85 \%$; $\mathrm{P}<0.0001)$. Importantly, high-dose CLB was significantly more effective in reducing drop seizure rates compared with low-dose CLB $(\mathrm{P}<0.0001)$. Eighty-nine percent of responders in the high-dose group and $56 \%$ in the lowdose group experienced $\mathrm{a} \geq 25 \%$ reduction in drop seizures $(\mathrm{P}=0.0025) ; 83$ and $38 \%$ experienced $\mathrm{a} \geq 50 \%$ reduction $(\mathrm{P}=0.0001) ; 67$ and $25 \%$ experienced a $\geq 75 \%$ reduction $(\mathrm{P}=0.0006)$; and 6 and $22 \%$ experienced a $100 \%$ reduction $(\mathrm{P}=0.0629)$. The study showed that CLB reduces the non-drop seizure rates as well, particularly in the high-dose group. The percent change in the low-dose group was not significant, however in the high-dose group the percent change from baseline $(59 \pm 55 \%$, $\mathrm{n}=22$ ) was significant. The dose-dependent manner of reducing drop seizure rates was also recorded for non-drop seizure rates, the reduction being significantly greater in the high-dose group compared with the low-dose group $(\mathrm{P}=0.0222)$. Parent/caregiver and investigator global evaluations have both demonstrated that the high-dose CLB group showed significantly greater improvements in overall symptoms compared to low-dose CLB group. At one investigation site, the quality of life of four children receiving CLB was greatly improved, as they discontinued wearing helmets and therefore they were able to move freely with- 
out the constant adult supervision previously needed to prevent injury from drops. ${ }^{6}$

A more advanced phase III, double-blind, placebo-controlled study on the safety and efficacy of CLB in patients with LGS aged 2-54 was conducted at 51 sites in the United States, India, Europe and Australia between August 2007 and December 2009 and further assessed CLB's role as adjunctive therapy.7 This study evaluated the efficacy of 3 CLB dosages in decreasing weekly frequencies of drop and total seizures and also assessed its safety when administered $\geq 18$ weeks at these 3 dosages. Patients aged 2-60 years were eligible to participate if they had onset of LGS before 11 years age and currently weighed $\geq 12.5 \mathrm{~kg}$. The study included 4 -week baseline, 3 -week titration and 12-week maintenance periods, followed by either continuation in an openlabel study or a 2- or 3- week taper period. Patients were randomly assigned to one of 4 groups, depending on weight (12.5 kg to $\leq 30$ $\mathrm{kg},>30 \mathrm{~kg}$ ): i) placebo; ii) low-dosage CLB: target of $0.25 \mathrm{mg} / \mathrm{kg} /$ day (maximum, $10 \mathrm{mg} / \mathrm{day}$ ); iii) medium-dosage CLB: target of 0.5 $\mathrm{mg} / \mathrm{kg} /$ day (maximum, $20 \mathrm{mg} /$ day); or iv) highdosage CLB: target of $1.0 \mathrm{mg} / \mathrm{kg} /$ day (maximum, $40 \mathrm{mg} /$ day). The mean patient age was 12.4 years. Importantly, approximately $50 \%$ of all patients were receiving concomitant valproic acid, valproate semisodium, or valproate sodium.

The mean percentage decrease in average weekly rate of drop seizures from baseline to maintenance period was $12.1 \%$ for placebo $v s$ 41.2\% ( $\mathrm{P}=0.0120), 49.4 \%(\mathrm{P}=0.0015)$ and $68.3 \%(\mathrm{P}<0.0001)$ for the $0.25,0.50$ and 1 $\mathrm{mg} / \mathrm{kg} /$ day dosage groups respectively. Mean difference from the placebo group increasing with increasing CLB dosage (mean differences of $29.1,37.3$ and $56.1 \%$ for the low, medium and high respectively). A linear trend has been noted in that increasing dosage of CLB lead to increased efficacy in reducing the drop seizure rates $(\mathrm{P}<0.0001)$. The mean percentage decrease in average weekly rate of total (drop and non-drop) seizures was $9.3 \%$ for placebo vs $34.8 \%(\mathrm{P}=0.0414), 45.3 \%(\mathrm{P}=0.0044)$, and $65.3 \%(\mathrm{P}<0.0001)$ for the CLB 0.25-, 0.5-, and $1.0-\mathrm{mg} / \mathrm{kg} /$ day groups.

A $40.0 \%$ decrease in the average weekly rate of non-drop seizures was observed for the high-dosage group, however this was not statistically significant by analysis of covariance model. An increase of 76.3, 53.3 and $3.3 \%$ has been noted in the average weekly rate of nondrop seizures for the placebo, low-dosage and medium-dosage CLB group.

Increasing CLB dosage lead to increase response rates in patients with LGS. The percentage of patients with $\geq 50 \%$ decrease from baseline to maintenance period in average weekly rate of drop seizures was $31.6 \%$ for placebo, 43.4, 58.6 and $77.6 \%$ for the low-, medium-, and high-dosage CLB groups, respectively. In comparison with the placebo group, the likelihood of achieving $\geq 50 \%$ response was greater for the medium-dosage and high-dosage CLB groups. Seizure-free patients have also been reported: 2 patients in the placebo group (3.5\%) were seizure-free, compared with 4 (7.5\%), 7(12.1\%) and 12 (24.5\%) patients for the low-, medium-, and high-dosage CLB groups.

Global evaluations of patients' overall changes in symptoms from physicians and caregivers during the observed period have also shown the CLB as adjunctive therapy led to improvements: percentages of patients who were at least minimally improved range from 71.2 to $80.7 \%$ (physicians' assessments) and 79.2 to $81.6 \%$ (caregivers' assessments) for CLB vs 47.3 and $45.5 \%$ respectively for placebo.

Although retrospective studies are statistically less significant than randomized controlled trials, they still provide valuable information about the effectiveness and safety of CLB in patients with epilepsy. A retrospective study was conducted between January 2013 and January 2015 in patients suffering from status epilepticus (SE). SE is defined as seizures lasting $>5$ min or multiple seizures without recovery of consciousness in between. Refractory status epilepticus (RSE) is defined as SE that persists despite adequate treatment with benzodiazepines and at least one AED, or $\mathrm{SE}$ requiring general anesthesia. About 12$43 \%$ of the cases with SE become refractory, and $50 \%$ of those requiring anesthesia will become super-refractory. Patients from all age groups in whom CLB was administered for the management of SE were included in the study. Over a period of 24 months, 17 patients received CLB for the treatment of RSE. In all these patients, CLB was used as add-on therapy after failure of two or more AEDs in adequate dosing and it was the last AED added in $94 \%$ of the patients. Thirteen patients reported a successful response to CLB (76.5\%). ${ }^{8}$

Another retrospective study conducted at the Hospital de Clínicas da Unicamp on 97 patients ranged 15 to 70 years who were evaluated for surgery and had been followed-up for $\geq 1$ year has evaluated the effectiveness of CLB as add-on therapy. Of these 97 patients, $74.2 \%$ had temporal lobe epilepsy, $8.2 \%$ had extratemporal epilepsy and in $17.6 \%$ patients the epileptic syndrome could not be identified. CLB was introduced after previous failure of at least two mono-therapies, with carbamezapine, phenytoin or valproate used up to maximum tolerated dose. The dosage of CLB ranged from 10 to $60 \mathrm{mg}$ twice a day and the period of usage ranged from 1 month to 7 years and 9 months. The study brought proof to the effectiveness of CLB as adjunctive therapy: 7.2\% patients were seizure-free, $49.4 \%$ had $\geq 50 \%$ improvement in seizure control and $40.2 \%$ patients had $<50 \%$ improvement in seizure control. In $3.1 \%$ no data were available. ${ }^{9}$

A review study conducted in 2011 evaluated several studies on the effectiveness of CLB, 10 both prospective and retrospective studies. In pediatric patients with refractory epilepsy, six open-label prospective studies have shown that at least $54 \%-85 \%$ of patients experienced at least a $50 \%$ drop in seizure rates (Table 1). $6,7,11-20$

Additionally, two retrospective studies have also reported significant decrease in seizure rates for pediatric patients using CLB as addon therapy (Table 1).11,17

Clinical studies of LGS were identified in a 2009 Cochrane review and by electronic database search and indirect comparison of the relative efficacies of CLB, felbamate, lamotrigine, topiramate and rufinamide as adjunctive treatments for LGS was performed. These indirect comparisons were performed by transforming the primary efficacy endpoint from each trial into Cohen's d effect size. The results have also shown that high-dosage CLB (1.0 $\mathrm{mg} / \mathrm{kg} /$ day) was the most effective $v s$ placebo, whereas medium-dosage CLB $(0.5 \mathrm{mg} / \mathrm{kg} / \mathrm{day})$ and rufinamide had moderate effects. Felbamate, lamotrigine and topiramate had low effect sizes. Numbers of total seizures and tonic-atonic seizures (drop attacks) were indirectly compared and both comparisons proved that medium- and high-dosage CLB are superior to the other adjunctive LGS therapies. ${ }^{21}$

A study that investigated potential drug interactions between CLB and other AEDs, including phenytoin, phenobarbital, carbamazepine, valproate, lamotrigine, felbamate, and oxcarbazepine, found no clinically meaningful drug pharmacokinetic interactions, which makes this drug suitable for the management of LGS as an adjunctive therapy due to its pharmacokinetic properties and less aggressive side effects. ${ }^{22}$

\section{Why should clobazam be pre- scribed by brand?}

The prescription of anti-epileptic medication can become an issue in the treatment of epilepsy. Practitioners are often encouraged to prescribe the cheapest drugs available and this is often inappropriate for the management of epilepsy. ${ }^{23} \mathrm{~A}$ research study conducted in 2003 suggests that even small differences between two versions of the same drug can become very problematic for the patient who is switching them. ${ }^{23}$ Crawford et al. suggest that these problems include additional side-effects or seizures frequency. ${ }^{24}$ However, the necessity of prescribing CLB by brand is a hypothesis based on the available evidence on other AEDs.

The claimed advantage of prescribing gener- 


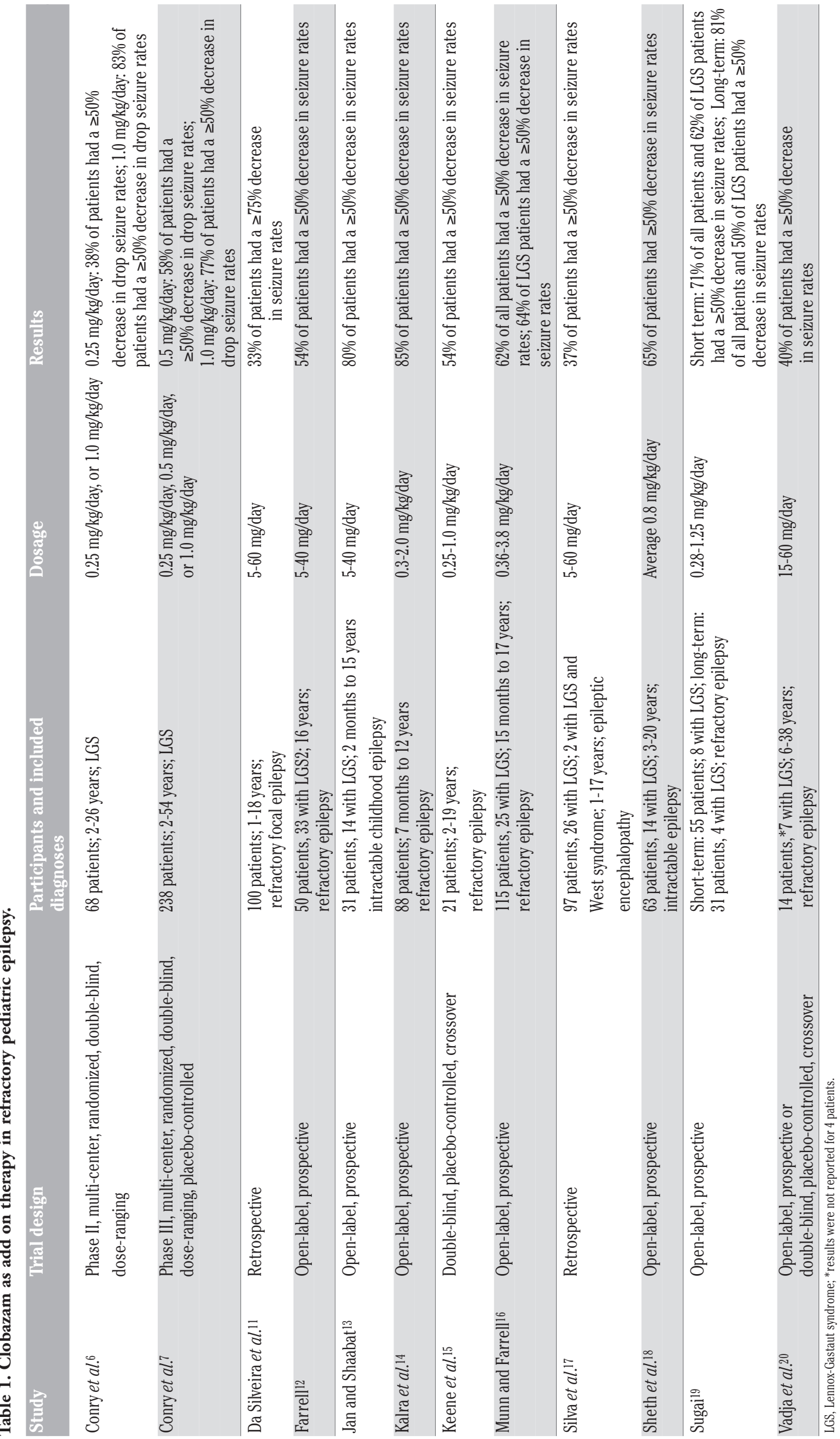


ically is that large amounts of money can be saved. However, the hidden consequences of generic prescribing is that costs may actually increase due to increased doctor visit (as a result of patient anxiety), increased sick leave, worse health for the patient and even in some cases potential loss of employment (Table 1). ${ }^{23}$

A survey of 1851 patients with epilepsy conducted by Epilepsy Action revealed that in the previous year, $33 \%$ of responders were given a different version of brand of their regular AED. Of these, almost 25\% experienced an increase in seizure frequency as a result and $33 \%$ experienced more or distinct side effects from the ones previously experienced. ${ }^{23}$

The survey also showed that a significant number of people (24\%) reported that they received a variety of versions of their medication in one single prescription. 23

The recommended guideline from he National Institute for Health and Care Excellence (NICE) with regards to this issue states as follows: Changing brand of AED is not recommended due to variances in bioavailability/ difference in pharmacokinetic profiles, which leads to increased potential for reduced effect or excessive side-effects (NICE, 2004). ${ }^{25}$

Patient's anxiety is a factor of major importance in epilepsy, as it can easily trigger seizures. Patients with changed medication may be anxious to take them, which in turn may lead to the loss of seizure control. Suffering a seizure after a long seizure-free period could have dramatic consequences on the well being of the patient, both in terms of the impact it has on his life but also in terms of the damage a seizure can cause itself. The impact that a slightly different version of an AED can have on a patient's life is to be taken into consideration and must not be underestimated. 25,26

\section{What patient groups may ben- efit most from a prescription of clobazam?}

The NICE recommends CLB as an adjunctive treatment where first-line antiepileptic drug have failed. ${ }^{27}$

NICE recommends CLB as an adjunctive treatment option for seizures: i) focal seizures; ii) generalized tonic-clonic seizures; and epilepsies: i) benign epilepsy with centrotemporal spikes; ii) Panayiotopoulous syndrome; iii) late-onset childhood occipital epilepsy (Gastaut type); iv) Dravet syndrome; v) epilepsy with generalized tonic-clonic seizures only.

NICE recommends CLB as an option on referral to tertiary care for seizures: i) generalized myoclonic seizures; ii) generalized absence seizures; and epilepsies: i) childhood absence epilepsy or other absence epilepsy syndromes; ii) juvenile absence epilepsy or other absence epilepsy syndromes; iii) juvenile myoclonic epilepsy; iv) idiopathic generalized epilepsy.

\section{What patient groups may ben- efit most from a licensed liquid formulation of clobazam?}

\section{Accurate Dosing}

\section{Children}

The ability to prescribe and administer safe and accurate doses of anti-epileptic drugs is fundamentally important in the treatment of epilepsy. The potential, however, for dosing accuracy in young children using CLB tablets is limited, as the smallest dose that can be accurately administered is $5 \mathrm{mg}$; it is for this reason that CLB tablets are not licensed for children under 6 years of age. Children between the age of 1 month and 6 years require small, weight-based doses, beginning at $125 \mathrm{mcg} / \mathrm{kg}$ twice a day. CLB oral suspension allows for the simple measurement of accurate doses, which will support compliance and offer the opportunity for optimal seizure control.

\section{Other patient groups}

CLB suspension may be beneficial for patients who require small starting doses of CLB (doses $<5 \mathrm{mg}$ ), for example elderly patients or in those known to be poor CYP 2C19 metabolizers.

\section{Ease of Administration}

Swallowing difficulties (or the inability to tolerate solid oral dosage forms) are over-represented in both adults and children with severe seizure disorders. Known risk factors for refractory epilepsy includes diffuse brain injury, genetic and metabolic disorders and underlying brain abnormalities, all of which are likely to be associated with additional neurological and/or behavioral deficit that may preclude, or at least complicate, dosing with solid oral dosage forms. Oral CLB suspension offers these patients a more acceptable formulation, which removes the need to crush tablets, saving patient/caregiver time, while potentially supporting compliance. The scientific evidence backing the administration of CLB orally is lacking, however this is a pragmatic recommendation that can benefit a lot of patients who have swallowing difficulties.

\section{Anxious patients}

While epilepsy represents the majority of CLB prescribing, it is also used in the shortterm treatment of anxiety in adults, where it is reserved for the management of anxiety that is severe, disabling or subjecting the individual to unacceptable distress. Although drug administration in this patient group might not pose the same complexity as is associated with the epileptic group this is, none-the-less, a severely unwell cohort for whom compliance may be compromised and for whom availability of CLB oral suspension is advantageous. This group is likely to include some elderly patients, for whom the possibility of using smaller doses would be desirable.

\section{References}

1. Gauthier AC, Mattson RH. Clobazam: a safe, efficacious, and newly rediscovered therapeutic for epilepsy. CNS Neurosci Ther 2015;21:543-8.

2. Giarratano M, Standley K, Benbadis SR. Clobazam for treatment of epilepsy. Expert Opin Pharmacother 2012;13:227-33.

3. Faulkner MA. Comprehensive overview: efficacy, tolerability, and cost-effectiveness of clobazam in Lennox-Gastaut syndrome. Ther Clin Risk Manag 2015;11:905-14.

4. Jensen HS, Nichol K, Lee D, Ebert B. Clobazam and its active metabolite $\mathrm{N}$ desmethylclobazam display significantly greater affinities for alpha(2)- versus alpha(1)-GABA(A)-receptor complexes. PloS One 2014;9:e88456.

5. Joshi R, Tripathi M, Gupta P, Gupta YK. Effect of clobazam as add-on antiepileptic drug in patients with epilepsy. Indian $\mathbf{J}$ Med Res 2014;140:209-15.

6. Conry JA, Ng YT, Paolicchi JM, et al. Clobazam in the treatment of LennoxGastaut syndrome. Epilepsia 2009;50:115866.

7. Ng YT, Conry JA, Drummond R, et al. Randomized, phase III study results of clobazam in Lennox-Gastaut syndrome. Neurology 2011;77:1473-81.

8. Sivakumar S, Ibrahim M, Parker D Jr, et al. Clobazam: an effective add-on therapy in refractory status epilepticus. Epilepsia 2015;56:e83-9.

9. Montenegro MA, Cendes F, Noronha AL, et al. Efficacy of clobazam as add-on therapy in patients with refractory partial epilepsy. Epilepsia 2001;42:539-42.

10. Leahy JT, Chu-Shore CJ, Fisher JL. Clobazam as an adjunctive therapy in treating seizures associated with LennoxGastaut syndrome. Neuropsychiatr Dis Treat 2011;7:673-81.

11. Silveira MR, Montenegro MA, Franzon RC, et al. Effectiveness of clobazam as add-on therapy in children with refractory focal epilepsy. Arq Neuropsiquiatr 2006;64:70510.

12. Farrell K. Benzodiazepines in the treat- 
ment of children with epilepsy. Epilepsia 1986;27:S45-52.

13. Jan MM, Shaabat AO. Clobazam for the treatment of intractable childhood epilepsy. Saudi Med J 2000;21:622-4.

14. Kalra V, Seth R, Mishra D, Saha NC. Clobazam in refractory childhood epilepsy. Indian J Pediatr 2010;77:263-6.

15. Keene DL, Whiting S, Humphreys $P$. Clobazam as an add- on drug in the treatmet of refractory epilepsy of childhood. Can J Neurol Sci 1990;17:317-9.

16. Munn R, Farrell K. Open study of clobazam in refractory epilepsy. Pediatr Neurol 1993;9:465-9.

17. Silva RC, Montenegro MA, Guerreiro CA, Guerreiro MM. Clobazam as add-on therapy in children with epileptic encephalopathy. Can J Neurol Sci 2006;33:209-13.

18. Sheth RD, Ronen GM, Goulden KJ, et al. Clobazam for intractable pediatric epilep- sy. J Child Neurol 1995;10:205-8.

19. Sugai K. Clobazam as a new antiepileptic drug and clorazepate dipotassium as an alternative antiepileptic drug in Japan. Epilepsia 2004;45:20-5.

20. Vajda FJ, Bladin PF, Parsons BJ. Clinical experience with clobazam: a new 1,5 benzodiazepine in the treatment of refractory epilepsy. Clin Exp Neurol 1985;21:177-82.

21. Cramer JA, Sapin C, Francois C. Indirect comparison of clobazam and other therapies for Lennox-Gastaut syndrome. Acta Neurol Scand 2013;128:91-9.

22. Walzer M, Bekersky I, Blum RA, Tolbert D. Pharmacokinetic drug interactions between clobazam and drugs metabolized by cytochrome P450 isoenzymes. Pharmacotherapy 2012;32:340-53.

23. Goodwin, M. The importance of brand continuity in epilepsy drugs. Nurs Times 2005;101:26-7.
24. Crawford P, Hall WW, Chappell B, et al. Generic prescribing for epilepsy. Is it safe? Seizure 1996;5:1-5.

25. [No authors listed]. Generic prescribing in epilepsy. Evid Based Med 2010;15:65-7.

26. Di Bonaventura C, Fattouch J, Fabbrini G, et al. Switching from branded to generic antiepileptic drugs as a confounding factor and unpredictable diagnostic pitfall in epilepsy management. Epileptic Disord 2007;9:465-6.

27. National Institute for Health and Clinical Excellence. The epilepsies: the diagnosis and management of the epilepsies in adults and children in primary and secondary care: pharmacological update of clinical guideline 20. London, Royal College of Physicians (UK): National Clinical Guideline Centre; 2012. 